\title{
CYLINDRICAL HARDY TYPE INEQUALITIES WITH BESSEL PAIRS
}

\author{
NGUYen TUAN DUY AND LE LONG PHI*
}

Abstract. Using factorizations of suitable partial differential operators and the notion of Bessel pairs, we establish several cylindrical Hardy's type identities and inequaltities in the sense of Badiale-Tarantello [2].

Mathematics subject classification (2020): Primary 26D10, 35A23, 46E35.

Keywords and phrases: Bessel pair, Hardy inequality, best constant.

\section{REFERENCES}

[1] Adimurthi, N. Chaudhuri, M. Ramaswamy, An improved Hardy-Sobolev inequality and its application, Proc. Amer. Math. Soc. 130 (2002), no. 2, 489-505.

[2] M. Badiale, G. TARAntello, A Sobolev-Hardy inequality with applications to a nonlinear elliptic equation arising in astrophysics, Arch. Ration. Mech. Anal. 163 (2002), no. 4, 259-293.

[3] A. A. BAlinsKy, W. D. Evans, R. T. LeWIS, The analysis and geometry of Hardy's inequality, Universitext. Springer, Cham, 2015. xv+263 pp.

[4] G. Barbatis, S. Filippas, A. Tertikas, A unified approach to improved $L^{p}$ Hardy inequalities with best constants, Trans. Amer. Math. Soc. 356 (2004), no. 6, 2169-2196.

[5] W. Beckner, Pitt's inequality and the fractional Laplacian: sharp error estimates, Forum Math. 24 (2012), no. 1, 177-209.

[6] G. Bertin, Dynamics of galaxies, Cambridge University Press, Cambridge, 2000.

[7] H. BRezis, J. L. VÁzQueZ, Blow-up solutions of some nonlinear elliptic problems, Rev. Mat. Univ. Complut. Madrid, 10, 1997, 443-469.

[8] L. CiotTi, Dynamical models in astrophysics, Scuola Normale Superiore, Pisa, 2001.

[9] N. T. DUY, N. LAM, P. LE, Improved Hardy inequalities and weighted Hardy type inequalities with spherical derivatives, Rev. Mat. Complut. (2020). https://doi.org/10.1007/s13163-020-00379-3.

[10] N. T. DuY, N. LAM, P. LE, Some Hardy identities on half-spaces, to appear in Math. Nachr.

[11] N. T. Duy, N. LAM, N. Triet, Hardy-Rellich identities with Bessel pairs, Arch. Math. (Basel) 113 (2019), no. 1, 95-112.

[12] N. T. DuY, N. LAM, N. TRIET, Hardy and Rellich inequalities with exact missing terms on homogeneous groups, J. Math. Soc. Japan 71 (2019), no. 4, 1243-1256.

[13] N. T. DuY, N. LAM, N. TRIET, Improved Hardy and Hardy-Rellich type inequalities with Bessel pairs via factorizations, J. Spectr. Theory 10 (2020), no. 4, 1277-1302.

[14] S. Filippas, V. MaZ'ya, A. TertiKas, Critical Hardy-Sobolev inequalities, J. Math. Pures Appl. (9) 87 (2007), no. 1, 37-56.

[15] F. Gazzola, H.-C. GRUnAU, E. Mitidieri, Hardy inequalities with optimal constants and remainder terms, Trans. Amer. Math. Soc. 356 (2004), no. 6, 2149-2168.

[16] F. Gesztesy, L. L. Littlejohn, Factorizations and Hardy-Rellich-type inequalities, Non-linear partial differential equations, mathematical physics, and stochastic analysis, 207-226, EMS Ser. Congr. Rep., Eur. Math. Soc., Zürich, 2018.

[17] F. Gesztesy, L. L. Littlejohn, I. Michael, M. Pang, Radial and logarithmic refinements of Hardy's inequality, reprinted in St. Petersburg Math. J. 30 (2019), no. 3, 429-436. Algebra i Analiz 30 (2018), no. 3, 55-65.

[18] F. Gesztes y, L. L. Littlejohn, I. Michael, R. Wellman, On Birman's sequence of HardyRellich-type inequalities, J. Differential Equations 264 (2018), no. 4, 2761-2801. 
[19] F. Gesztesy, L. Pittner, A generalization of the virial theorem for strongly singular potentials, Rep. Math. Phys. 18, 149-162 (1980).

[20] N. Ghoussoub, A. Moradifam, Bessel pairs and optimal Hardy and Hardy-Rellich inequalities, Math. Ann. 349 (2011), no. 1, 1-57.

[21] N. Ghoussoub, A. Moradifam, Functional inequalities: new perspectives and new applications, Mathematical Surveys and Monographs, 187, American Mathematical Society, Providence, RI, 2013. xxiv +299 .

[22] N. Ghoussoub, A. Moradifam, On the best possible remaining term in the Hardy inequality, Proc. Natl. Acad. Sci. USA 105 (2008), no. 37, 13746-13751.

[23] J. A. Goldstein, I. Kombe, A. Yener, A unified approach to weighted Hardy type inequalities on Carnot groups, Discrete Contin. Dyn. Syst. 37 (2017), no. 4, 2009-2021.

[24] N. IOKU, M. Ishiwata, T. OZAWA, Sharp remainder of a critical Hardy inequality, Arch. Math. (Basel) 106 (2016), no. 1, 65-71.

[25] A. Kufner, L. Maligranda, L.-E. Persson, The Hardy Inequality. About its History and Some Related Results, Vydavatelský Servis, Pilsen, 2007.

[26] A. KufNer, L.-E. Persson, N. SAmko, Weighted inequalities of Hardy type second edition, World Scientific Publishing Co. Pte. Ltd., Hackensack, NJ, 2017. xx+459 pp.

[27] N. LAM, A note on Hardy inequalities on homogeneous groups, Potential Anal. 51 (2019), no. 3, 425-435.

[28] N. LAm, Hardy and Hardy-Rellich type inequalities with Bessel pairs, Ann. Acad. Sci. Fenn. Math. 43 (2018), 211-223.

[29] N. LAm, G. Lu, L. ZHANG, Factorizations and Hardy's type identities and inequalities on upper half spaces, Calc. Var. Partial Differential Equations 58 (2019), no. 6, Art. 183, 31 pp.

[30] N. LAM, G. LU, L. ZHANG, Geometric Hardy's inequalities with general distance functions, J. Funct. Anal. 279 (2020), no. 8, 108673, 35 pp.

[31] S. Machihara, T. Ozawa, H. WADAde, Remarks on the Hardy type inequalities with remainder terms in the framework of equalities, In Asymptotic Analysis for Nonlinear Dispersive and Wave Equations, pp. 247-258. Mathematical Society of Japan, 2019.

[32] È. Mitidieri, A simple approach to Hardy inequalities (Russian), Mat. Zametki 67 (2000), no. 4, 563-572; translation in Math. Notes 67 (2000), no. 3-4, 479-486.

[33] V. H. NGuYen, New sharp Hardy and Rellich type inequalities on Cartan-Hadamard manifolds and their improvements, Proc. Roy. Soc. Edinburgh Sect. A, 1-30. doi:10.1017/prm.2019.37.

[34] B. OpIC, A. Kufner, Hardy-type inequalities, Pitman Research Notes in Mathematics Series, 219. Longman Scientific \& Technical, Harlow, 1990. xii+333 pp.

[35] M. Ruzhansky, B. Sabitbek, D. Suragan, Subelliptic geometric Hardy type inequalities on half-spaces and convex domains, Ann. Funct. Anal. 11 (2020), no. 4, 1042-1061.

[36] M. Ruzhansky, D. Suragan, Hardy inequalities on homogeneous groups, Progress in Math., Vol. 327, Birkhäuser, 2019, 588 pp.

[37] M. RuZhansky, N. YessiRKegenov, Factorizations and Hardy-Rellich inequalities on stratified groups, J. Spectr. Theory 10 (2020), no. 4, 1361-1411.

[38] M. Sano, F. TaKahashi, Scale invariance structures of the critical and the subcritical Hardy inequalities and their improvements, Calc. Var. Partial Differential Equations 56 (2017), no. 3, Art. 69 , $14 \mathrm{pp}$.

[39] S. Secchi, D. Smets, M. Willem, Remarks on a Hardy-Sobolev inequality, C. R. Math. Acad. Sci. Paris 336 (2003), no. 10, 811-815.

[40] J. L. VÁZQUEZ, E. ZUAZUA, The Hardy inequality and the asymptotic behaviour of the heat equation with an inverse-square potential, J. Funct. Anal. 173 (2000), no. 1, 103-153.

[41] Z.-Q. WANG, M. ZHU,, Hardy inequalities with boundary terms, Electron. J. Differential Equations 2003, No. 43, 8 pp. 